\title{
Light and ultrastructural relationship between oxytalan fibers in the periodontal ligament of the guinea pig
}

\author{
R. S. Edmunds, T. A. Simmons, C. F. Cox And J. K. Avery \\ Laboratory of Oral Histology, Dental Research Institute, \\ The University of Michigan, AnnArbor, Michigan, USA
}

\begin{abstract}
The interfaces and the relationships between collagen and oxytalan fibers were observed under light and electron microscopy. Guinea pig periodontal ligament was prepared for light and electron microscopy with perfusion using Peter's buffered formalin for light microscopy and GTA-Scollidine and $\mathrm{O}_{\mathrm{s}} \mathrm{O}_{4}$ for electron microscopic studies. The tissue for light microscopy was stained with a modified Gomori's aldehyde fuchsin technique, in which pre-oxidization with potassium monopersulfate was carried out before staining so as to demonstrate the oxytalan fibers. EM tissues were routinely stained with lead citrate and uranyl acetate.

Two different structural relationships were observed. First, the subcomponents of the collagen and oxytalan fiber types interweave with each other; and, second, some of these two-fiber subcomponents appear attached to each other. These relationships and the known orientation of oxytalan fibers as seen in the periodontal ligament provide insight as to the function of oxytalan fibers. The oxytalan fibers may provide increased structural integrity and increased distribution of forces over a wider area of the periodontal ligament. Because of their close relationship to blood and lymph vessels in the periodontal ligament, they may also help to stabilize these elements by the same structural relationships to collagen fibers.
\end{abstract}

Accepted for publication 7 August 1978

Since the original description of oxytalan fibers by Fullmer \& Lillie (1958), much research has been conducted in an attempt to better define the structure and function of this newly found connective tissue fiber type. The orientation, distribution in tissues, and ultrastructure of oxytalan fibers are now fairly well established. Current research, however, leaves the question of the function of these fibers unanswered. In order to fully grasp the concepts presented in the literature review and the results of research, a review of pertinent terminology is in order. Collagen fibers as observed under light microscopy are composed of many parallel collagen fibrils, which can be seen clearly under electron microscopy. The fibril is composed of even smaller procollagen subunits $(14 \times 2800 \AA)$, which impart the classic $640 \AA$ banding to the collagen fibril. Oxytalan fibers are composed of a number of subunits called filaments. A substructure to oxytalan filaments has not yet been demonstrated. (See Fig. 3 for illustra-

This work was supported by a BRS Grant \#5507-RR-05321-15. 
tion of fibers and components.)

Oxytalan fibers were originally observed in periodontal ligament after a staining sequence designed to demonstrate differences in stainability of collagen fibers at different ages, and in areas of stress (Fullmer 1958). It was also reported that oxytalan fibers could be observed in blood vessels, tendons, ligaments, connective tissue of hair follicles, epineurium and perineurium of human tissues. Further research has revealed that current histochemical methods for light microscopic study of these fibers do not demonstrate staining differences between oxytalan and pre-elastic fibers (Fullmer et al. 1974). The presence of oxytalan fibers has also been suggested in the tooth pulp (Fullmer 1959, Provenza et al. 1967) and in the tympanic membrane of humans (Cameron et al. 1970, Dawes 1970). However, these findings are based only on observations using light microscopy and current staining techniques. It has been suggested that existence of oxytalan fibers in nonperiodontal ligament tissues must be established by electron microscopy (Fullmer et al. 1974).

Along with the description of oxytalan fibers in normal tissues, their presence has also been noted in some pathologic tissues. Apparently typical oxytalan fibers, shown by light histology, were found in giant cell tumors of tendon sheaths, dermal fibromas (Tedeschi \& Sommers 1962), and sclerosing hemangiomas of skin (Tedeschi \& Sommers 1961). Oxytalan fibers have also been reported in dental granulomas, radicular cysts (Fullmer 1960) and periodontal membrane involved in scleroderma (Fullmer \& Witte 1962).

Sims (1977) described the presence of increased amounts of oxytalan staining fibers in the periodontal ligament of lathyritic mice. This fiber-like material increased in quantity as the degree of lathyrism increased. The lathritic material was demonstrated in sections oxidized and stained with orcein or aldehyde fuchsin. However, the author also suggested that the lathyritic material might be some form of lathyritic collagen because of its fiber-like appearance and its orientation, similar to major collagen bundles in the periodontal ligament.

The presence of oxytalan fibers has been described in the periodontal ligament over a range of species, including man, monkey, guinea pig, rat and mouse (Provenza 1965, Goggins 1966, Carmichael 1968). There may, however, be a species difference in the orientation of the fibers. In human periodontium the oxytalan fibers are described as following the course of the collagen fibers (Fullmer et al. 1974). In mice, the oxytalan fibers appear to be almost at right angles to the collagen fibers (Carmichael 1968, Sims 1973). The cervical areas of all species, however, display the largest size fibers and their arrangement is similar to normal collagen fibers. Most authors (Goggins 1966, Carmichael 1968, Sims 1973, Fullmer et al. 1974) agree that oxytalan fibers do insert into cementum and tend to aggregate around blood vessels. Some authors describe the fibers inserting into bone (Fullmer et al. 1974) while Sims (1973) has found no evidence to support this.

Oxytalan fibers at an orientation of right angles to the principal collagen fibers are in a position to provide increased mechanical strength to periodontal ligament if they are bonded or closely related to the collagen fibers. Such a theory of increased structural integrity and distribution of forces over a larger area of the periodontal ligament has beet proposed (Shakleford 1974). This theory describes the "Indifferent Fiber Plexus" in the periodontal ligament. This plexus is 


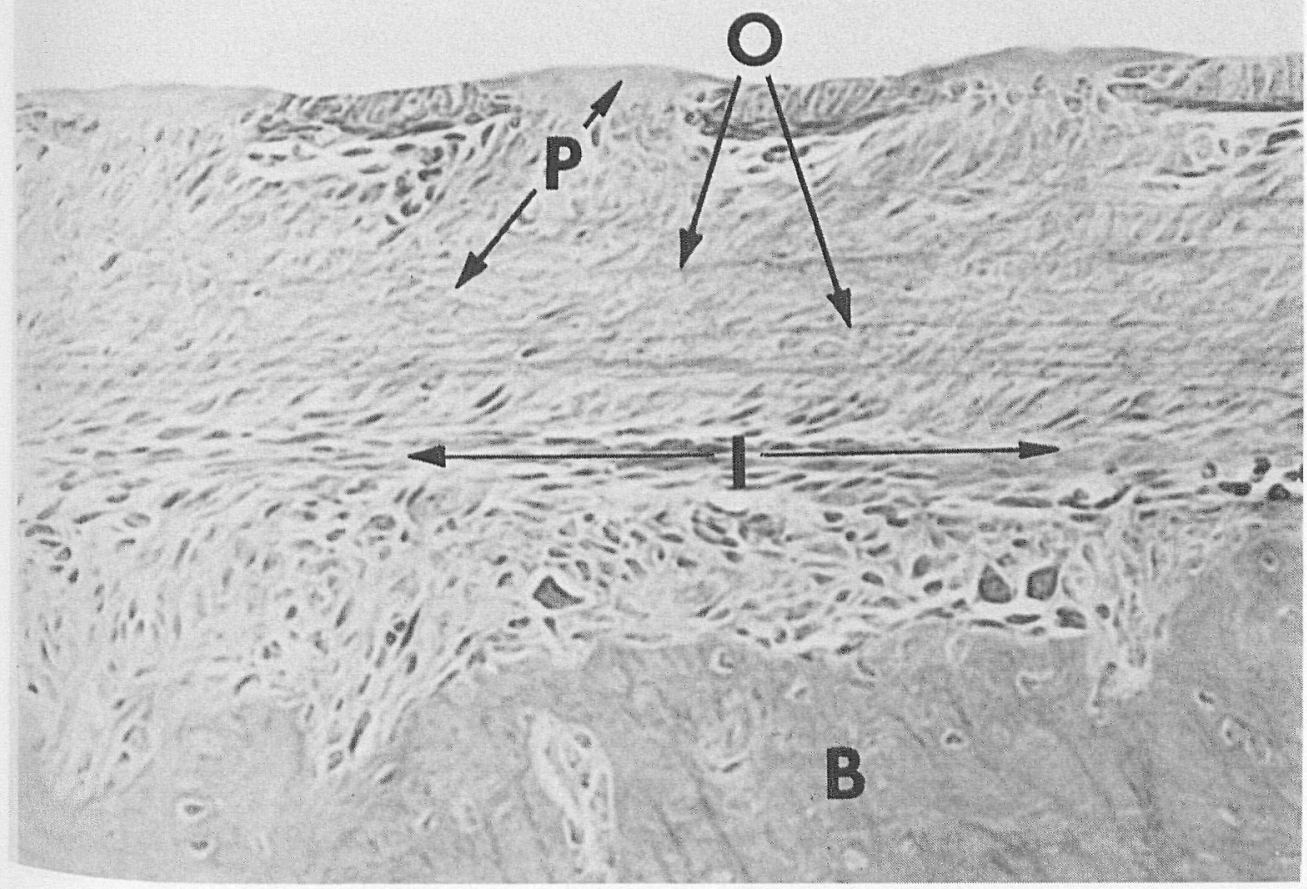

Fig. 1. Photomicrograph demonstrating the appearance of periodontal ligament from a guinea pig using modified Gormori's aldehyde fuchsin stain. Tissue present includes alveolar bone (B), principal collagen fibers $(\mathrm{P})$, oxytalan fibers $(\mathrm{O})$, indifferent fiber plexus $(\mathrm{I}) . \times 300$.

formed by interweaving and anastomosing of many collagen fibrils to the principal fiber. This plexus of collagen would distribute forces applied to the tooth over a larger area of the periodontal ligament.

Oxytalan fibers may be described as a separate type of connective tissue element because they may easily be distinguished from other connective tissue elements including collagen (Fullmer \& Lillie 1958), and reticulin fibers (Miles 1967). The resemblance of oxytalan to elastic fibers is founded on the observation that, in some animals in which the periodontal ligament contains elastic fibers, the orientation and distribution of elastic fibers is similar to that of oxytalan fibers found in human periodontal ligament (Fullmer et al. 1974). In enzymatic digestion experiments, Fullmer \& Lillie (1958) reported that the stainable portion of oxytalan fibers was digested by elastase only after prior oxidation with peracetic acid. This oxidation step is necessary to permit the staining of the fibers by histochemical methods as described below.

At the electron microscopic level, oxytalan fibers have a substructure composed of many fibrils which run parallel to the axis of the main fiber (Fullmer et al. 1974). These fibrils are about $150 \AA$ in diameter (Carmichael \& Fullmer 1966, Sheetz et al. 1973). Oxytalan fibers may also display a 


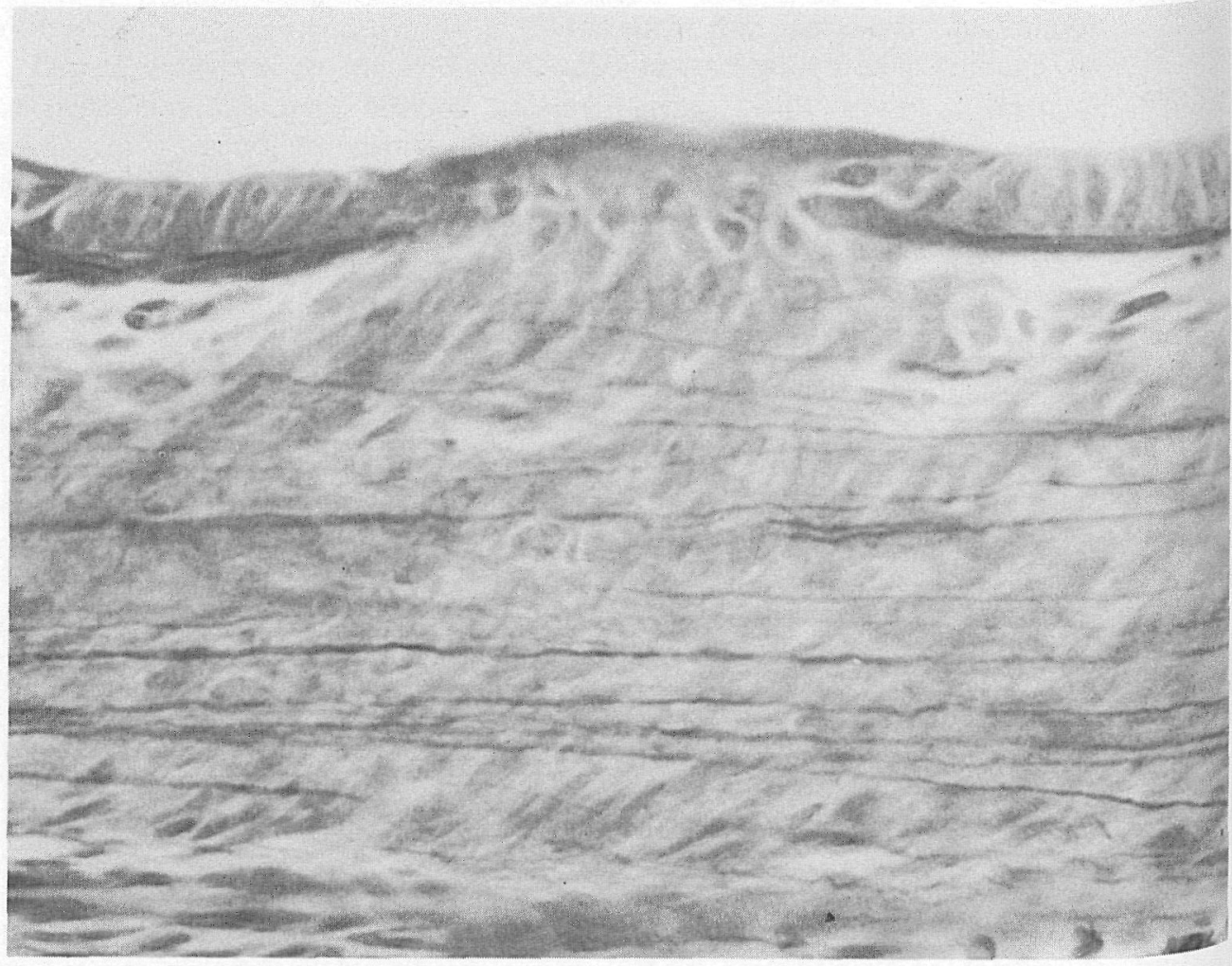

Fig. 2. Guinea pig periodontal ligament at higher magnification demonstrating branching and anastomosing of oxytalan fibers, principal collagen fibers and collagen fiber plexus. The orientation of the dark staining oxytalan fibers is at right angles to the principal collagen fibers. $\times 600$.

variable amount of interfibrillar amorphous material. A distinguishing observation of mature oxytalan fibers is that the fibrils are observed even in mature fibers (Fullmer et al. 1974). The substructure of immature elastic fibers resembles that of oxytalan fibers. However, in mature elastic fibers the amorphous material, elastin, accumulates to such a degree that it obscures the fibrils when observed under EM (Ross \& Bornstein 1969). Oxytalan fibers may be distinguished from elastic fibers morphologically at the electron microscopic level (Sheetz et al. 1973).

The presence of oxytalan fibers in light microscopy is demonstrable with the use of elastic tissue strains after oxidation with peracetic acid (Fullmer \& Lillie 1958) of with Oxone ${ }^{\circledR}$ (Rannie 1963). Oxone ${ }^{\circledR}$ is a bleach with potassium monopersulfate as the active ingredient.

The most commonly used histochemical staining procedure for oxytalan fibers at present is the Oxone ${ }^{\circledR}$-aldehyde fuchsin-Halmi method (Fullmer et al. 1974). Care must be taken because the aldehyde fuchsin stain for the oxytalant fibers is a transient dye that may decompose in 1 to 2 weeks (Fullmer et al. (B) E. I. Dupont Co., Electro Chemicals Dept, Wilmington, Del. 19801, U.S.A. 


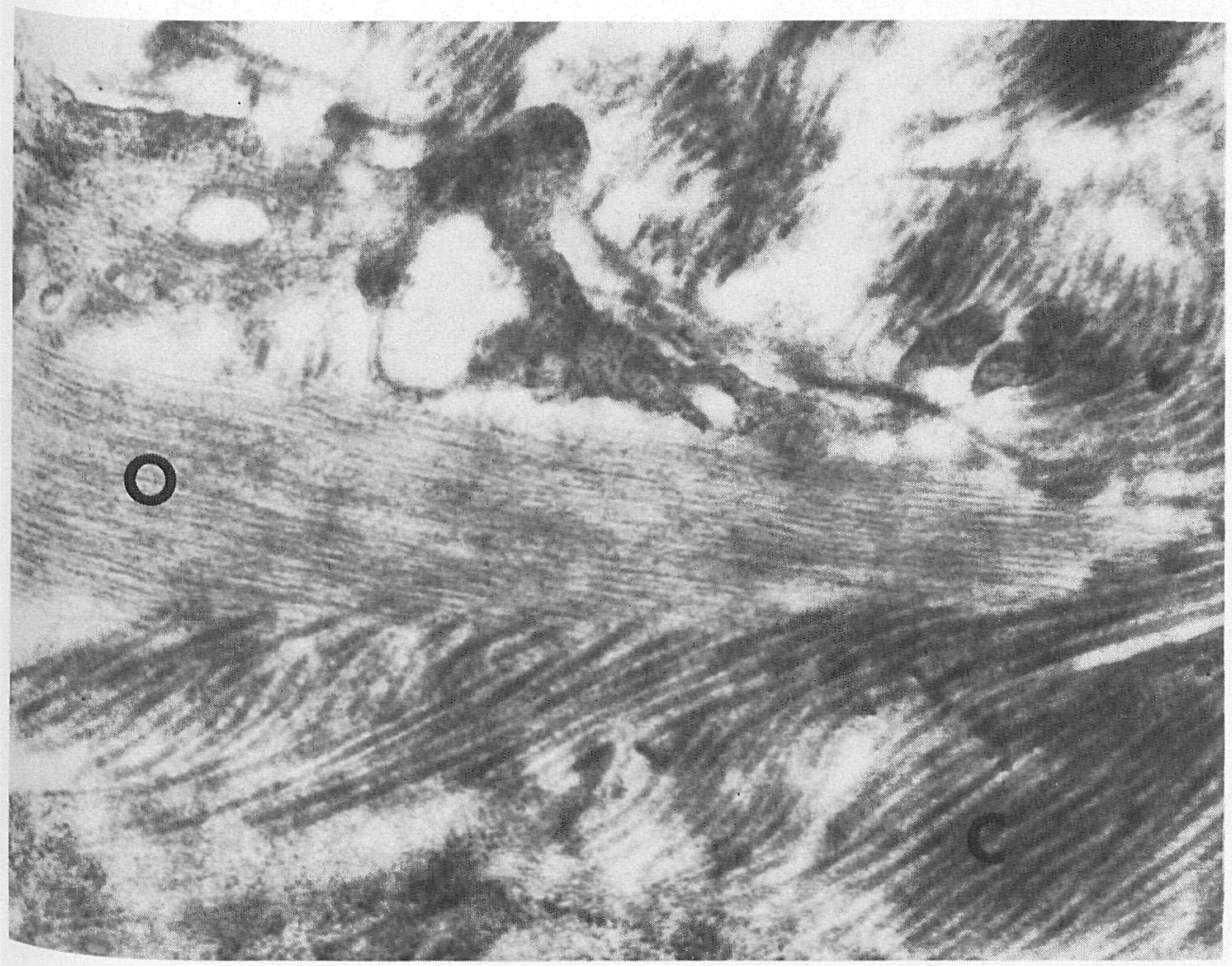

Fig. 3. Electron micrograph of collagen and oxytalan fibers in guinea pig periodontal ligament. The collagen fibrils (C) demonstrate $640 \AA$ banding, and many of these fibrils make up one collagen fiber. The oxytalan fiber $(O)$ is seen to be composed of many subunits, filaments, and demonstrates no amorphous substance as do elastic fibers. $\times 38,000$.

1974). With this method the oxytalan fibers appear purple, collagen cementum and dentin appear greenish yellow, and Soft tissue stains green.

Light microscopic studies have demonstrated that oxytalan fibers may contain an $\alpha$-hydroxyamine residue which, when oxidized by periodate, becomes imines. The imines undergo tautomerization to enamines which probably behave as the stainable nucleophilic species in the staining procedure (Mander et al. 1968).

Thus, the function of oxytalan fibers has not yet been determined. Several theories have been presented, but none has been clearly demonstrated.

(1) Observations by several authors suggest that teeth in firm occlusion and those under functional stress, such as bridge abutments, demonstrate more oxytalan fibers and that these fibers are larger than those on teeth not in function. These observations have led to the theory that oxytalan fibers play a role in response to functional stress (Fullmer \& Lillie 1958, Fullmer 1959, 1961, 1962, Fullmer et al. 1974).

(2) Beertsen et al. (1974) proposed a model for eruption of the rat incisor by suggesting that fibroblasts migrating in an occlusal direction, guided by oxytalan 


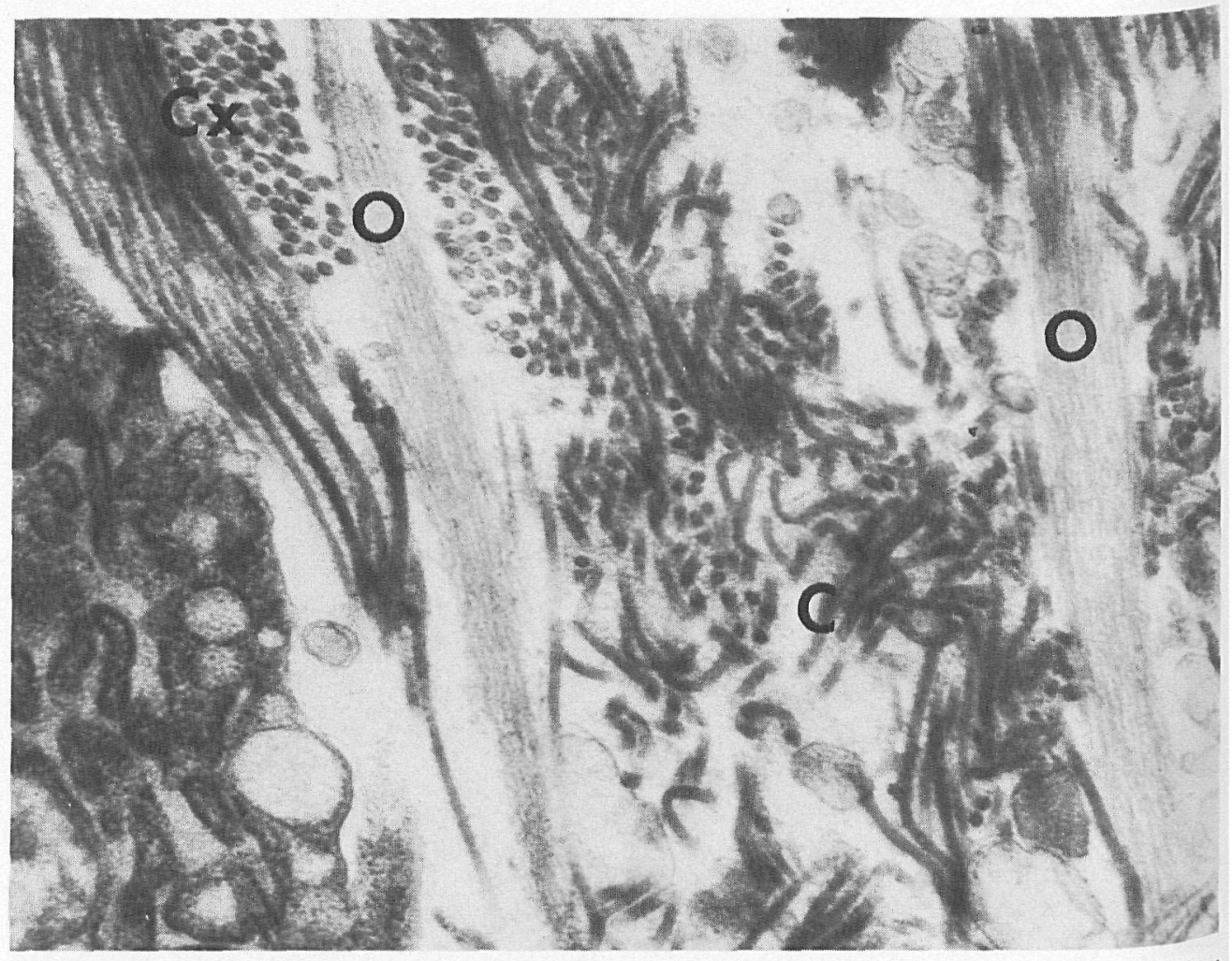

Fig. 4. EM micrograph demonstrating some of the collagen fibrils in cross section (CX), two longitudinal sections of oxytalan fibers are in the field $(O)$. This constitutes the right angle relationship seen in light microscopy. $\times 38,000$.

fibers, pull the tooth occlusally by means of collagen fibers.

(3) Fullmer et al. (1974) observed that oxytalan fibers adjacent to the necks of teeth in solid occlusion are more numerous and larger than in teeth out of occlusion. The same is true for teeth acting as abutments. These observations suggest that oxytalan fibers are physiologically responsive to functional stress.

(4) Edwards (1968) noted an increase in size and number of oxytalan fibers in the periodontal ligament of previously rotated teeth in beagle dogs. He theorized that oxytalan fiber may help to stop stretching of the periodontal tissues. Again, as with
Fullmer et al. (1974), more and larger fibers were noted in areas of functional stress, and again the local periodontal ligament was responsive and produced more and larger fibers.

(5) Sims (1973) reported the close relationship of oxytalan fibers to the blood vessels in the periodontal ligament. He points out that, as the tooth comes under different functional loads, the tension of oxytalan fibers connected to the tooth will change and thus may help to regulate the vascular flow in the periodontal vessels by this difference in tensional levels. Sims (1977) has modified the statement of this theory somewhat to state that the oxytalan 


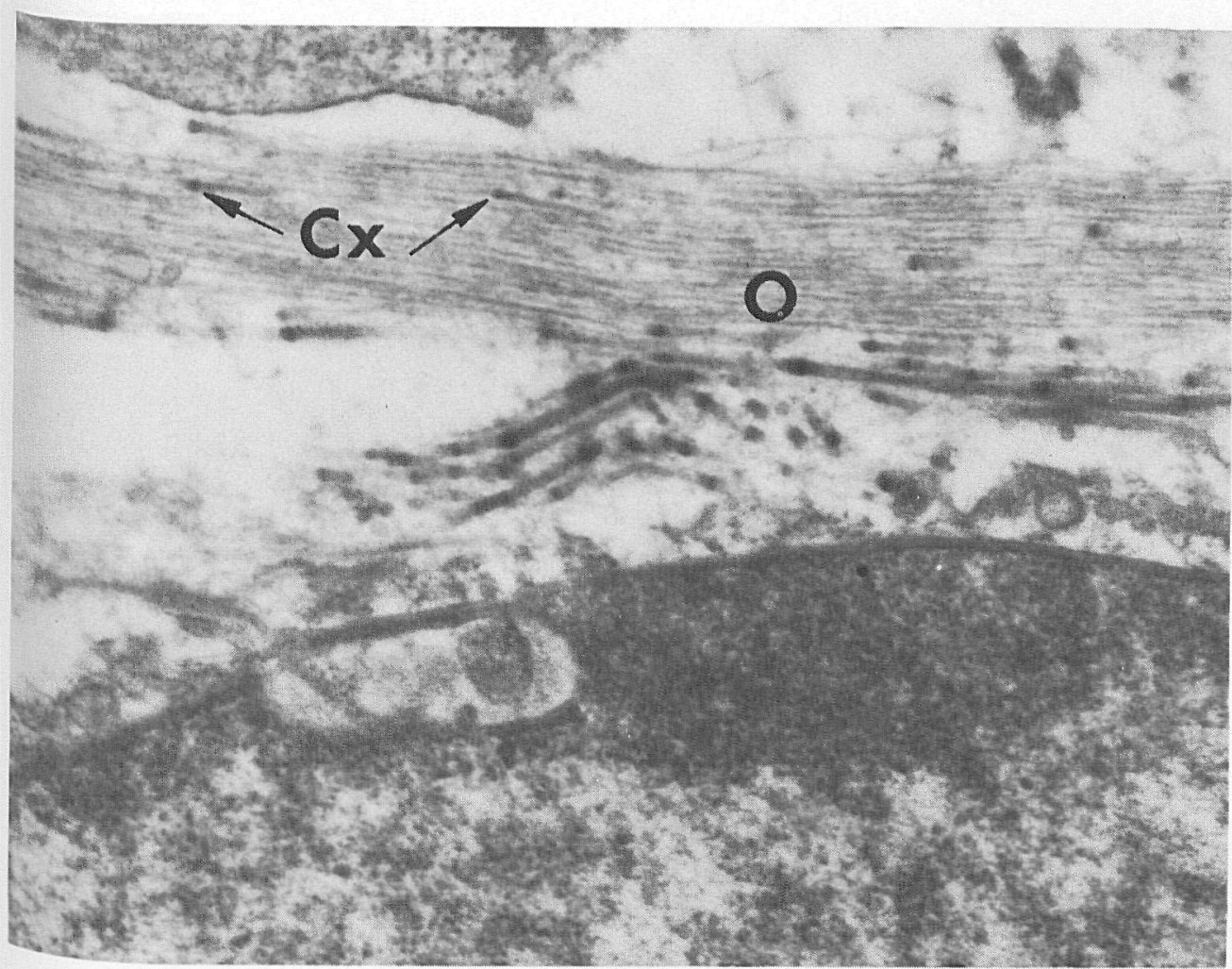

Fig. 5. EM micrograph demonstrating interweaving of the subcomponents of oxytalan and collagen fibers, oxytalan filaments, and collagen fibrils. Collagen fibrils are seen in cross section (CX), oxytalan fibers in longitudinal section (O). ×38,000.

fibers of the periodontal ligament may relate tooth movement to tensional changes in the vascular wall.

\section{Material and Methods}

TWO groups of young adult albino guinea pigs were used in this study, the first group for light microscopy and the second for electron microscopy. All animals were housed in individual cages and fed dry guinea pig diet and water ad libitum. The animals weighed approximately $500 \mathrm{~g}$ at the beginning of the study.

The group of animals used for light microscopy was sedated by ether inhalation and then intraperitoneally injected with $300 \mathrm{mg} / \mathrm{kg}$ of sodium pentobarbitol which induced complete anesthesia. The animals were perfused with $0.9 \%$ saline solution, followed with a wash of $10 \%$ phosphate buffered formalin (PBF). After $5 \mathrm{~min}$ of perfusate wash, the upper and lower molar teeth were removed, split into right and left sides and placed in vials of PBF for fixation. The lower incisors were removed and treated in the same fashion. After $48 \mathrm{~h}$ of fixation the specimens were rinsed in water and then decalcified with $0.5 \mathrm{M}$ ethylene diamine tetracetic acid (EDTA) and buffered to a $\mathrm{pH}$ of 7.2. The 


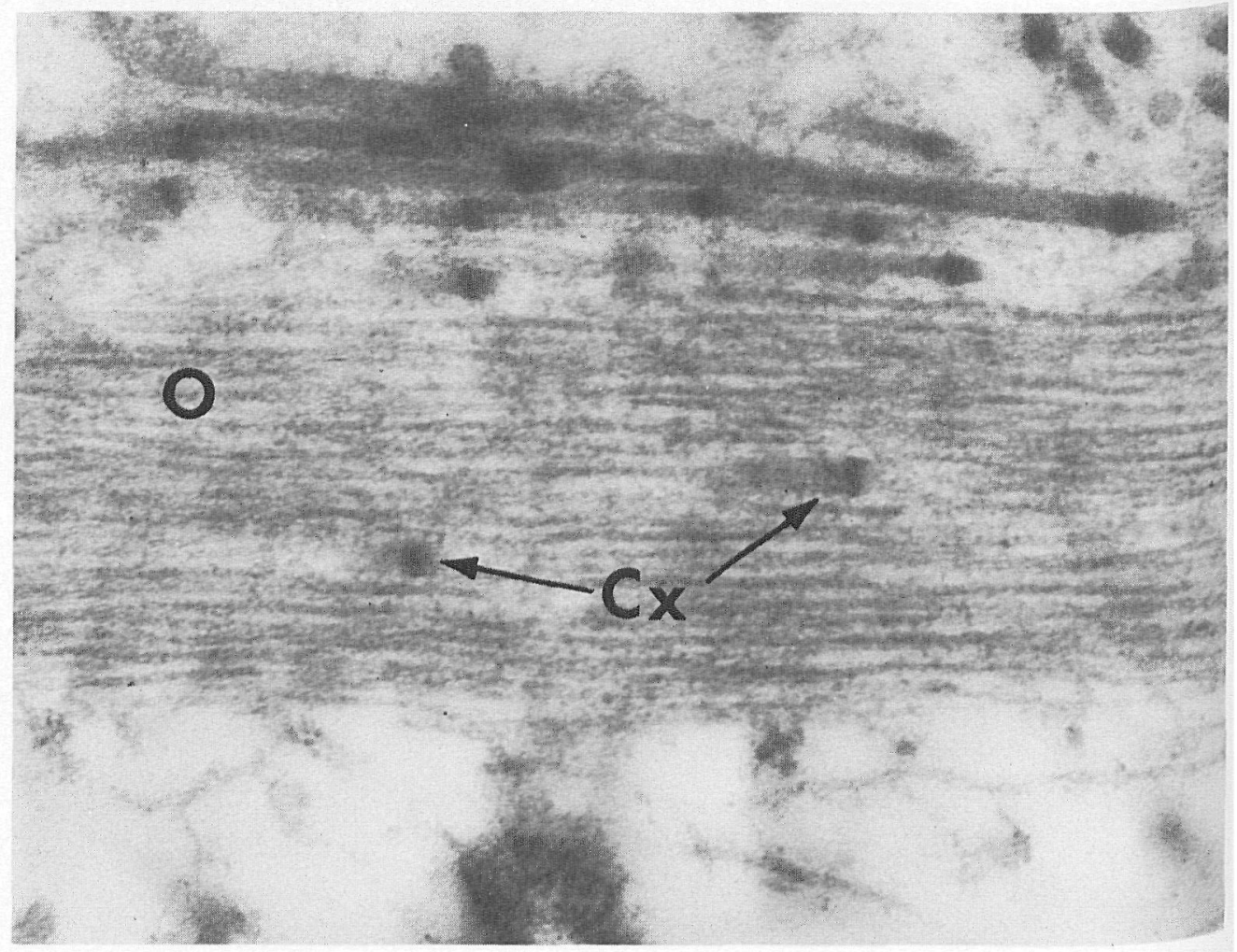

Fig. 6. Micrograph at a higher primary magnification more clearly demonstrates the interweaving of the two fiber types. Cross sections of collagen fibrils (CX), oxytalan fibers (O). $\times 98,000$.

decalcification process was monitored by taking radiographs of the tissue at intervals to determine the end point of chelation decalcification.

Upon complete decalcification, the tissue samples were dehydrated by a sequence of ethanol and butyl alcohols, infiltrated in paraplast and orientated en-bloc so that the buccal surface was parallel to the sectioning surface. Serial sections were taken at $8 \mu$ and mounted on gelatin subbed slides. Every third slide was stained with standard hematoxylin and eosin, Masson trichrome or Gomori's aldehyde fuchsin (Fullmer 1958).

The sequence of Gomori's aldehyde fuchsin was modified specifically to demonstrate oxytalan fibers. The central modification in this procedure is to oxidize the tissue in $10 \%$ aqueous Oxone ${ }^{\circledR}$ for $30 \mathrm{~min}$ allowing for the staining and visualization of the oxytalan fibers. Most of the tissue is oxidized in this step; however, the oxidized oxytalan fibers are stained with Gomori's aldehyde fuchsin and retain the stain after a differentiation step (Fullmer et al. 1974).

Observations at the light microscopic level were made on a Zeiss photomicroscope and pictures were taken.

The group of guinea pigs used for electron microscopy was prepared for perfusion in the same manner as the first group. They were first flushed with $0.9 \%$ 


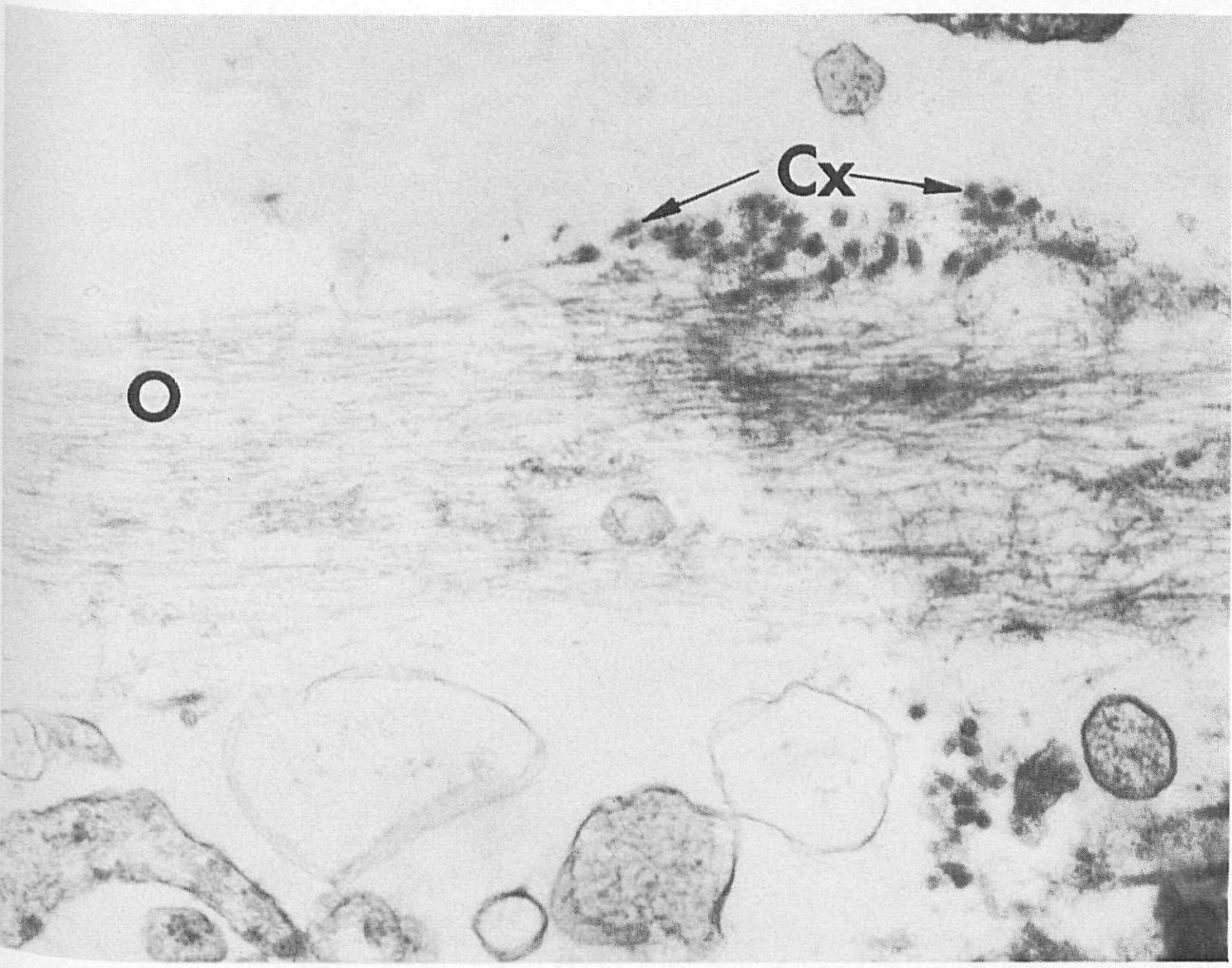

Fig. 7. EM micrograph demonstrating a diffuse oxytalan fiber $(\mathrm{O})$, and individual collagen fibrils in cross section (CX). The oxytalan filaments are very closely associated with the collagen fibrils. $\times 38,000$.

Saline, followed with a glutaraldehyde and S-collidine solution for perfusion fixation: The tissue samples were then removed and fixed in the solution of glutaldehyde and S-collidine for $2 \mathrm{~h}$. They were then washed in an S-collidine buffer and post-fixed in osmium tetroxide for $2 \mathrm{~h}$. At the end of the ethyl alcohol dehydration sequence, the tissues were then embedded in Epon ${ }^{\circledR}$ and polymerized. Thick $1 \mu$ trims were taken to confirm the tissue orientation and to aid in block trimming. Thin sections were then taken and placed on copper grids. The grids were stained with uranyl acetate and lead citrate. Observations were made using a Zeiss electron microscope and under a primary magnification of $\times 1000$ to $\times 25,000$.

\section{Results}

Observations made on the light microscopic level clearly demonstrated the presence of oxytalan fibers in the guinea pig periodontal ligament. The orientation was most frequently observed to be in an apical occlusal direction with the ends of the oxytalan fibers somewhat closer to the root structure than the apical ends. No oxytalan fibers were seen to insert into cementum or bone in this slide series (see Fig. 1). Principal collagen fibers were clearly observable as green fibers stained by the Halmi counterstain, and their orientation was from bone to cementum inserting into both. An interweaving and anastomosing plexus of collagen fibers and 


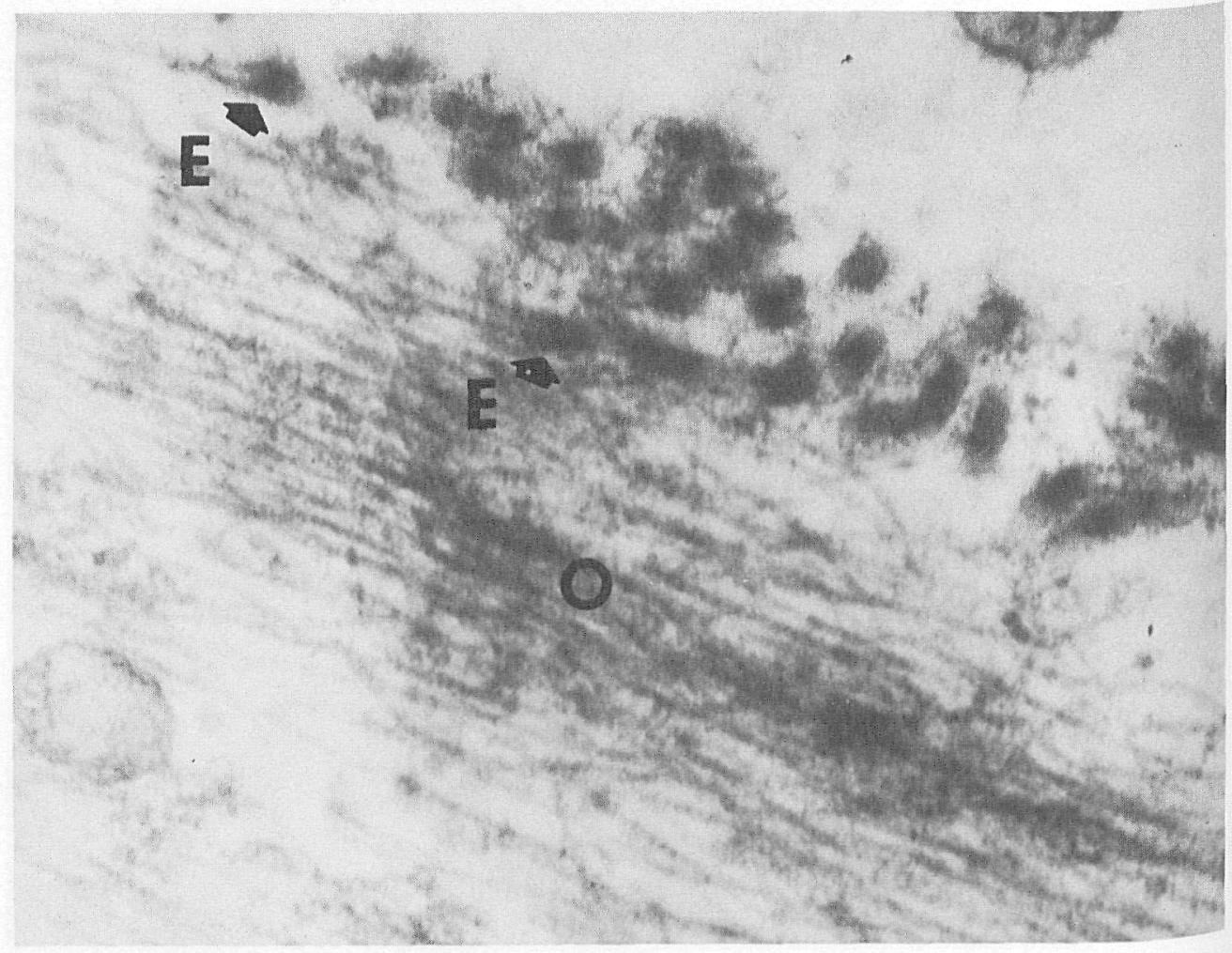

Fig. 8. EM micrograph demonstrating two areas where oxytalan filaments appear to end on collagen fibrils (E). The oxytalan fiber $(\mathrm{O})$ is at right angles to the collagen fibrils. $\times 98,000$.

interweaving collagen fibrils was also observed in an apical-cervical orientation.

At high magnification the interweaving and anastomosing plexus of collagen fibrils forming the "Indifferent Fiber Plexus" (Shackleford 1974) may again be observed (see Fig. 2). This higher magnification demonstrates the subcomponents of the oxytalan fibers, the oxytalan filaments, and the fact that they anastomose and interweave through the collagen much the same as do the collagen subunits.

At the EM level, collagen fibers and fibrils may be clearly seen. The fibril subunits exhibit the $640 \AA$ banding. When viewed in cross section, the collagen fibrils appear as round electron opaque dots. The oxytalan fibers are also visible and are seen to be composed of many electron dense lines which represent the filament substructure. It can be seen (Figs 3 and 4) how these subunits of both collagen and oxytalan branch from main fiber and rejoin to form other fibers.

The relationship of the two fiber types at their interfaces is demonstrated in Figs 5 and 6 . When viewing longitudinal sections of oxytalan fibers, cross sections of collagen fibrils are observed in the main portion of the oxytalan fiber. This indicates that the subcomponents of the two fiber types interweave with each other; collagen fibrils passing at $90^{\circ}$ to oxytalan fibers don't simply pass around the fiber, but pass through the oxytalan filament complex (oxytalan fiber). This relationship 
greatly increases the amount of contact surface area of the two fiber types.

The second relationship observed between the two fiber types is demonstrated in Figs 7 and 8. When longitudinal sections of oxytalan fibers were observed to be somewhat diffuse, as in Fig. 7, the individual oxytalan filaments may be seen more easily. When these diffuse fibers pass near collagen fibers, they appear in very close association. In some instances the oxytalan filaments seem to end directly on collagen fibrils. This is easily demonstrated when the collagen fibrils are in cross section (Figs 7 and 8).

\section{Discussion}

It is the opinion of the authors of the two structural observations that interweaving of the fiber subcomponents and connection of the two fiber types allows for an increased structural rigidity or turgor in the periodontal ligament, and distribution of forces over a larger area. It seems clear that, because the fiber subcomponents are so closely related, there is a resulting increase in contact surface area which makes many connections (more than previously thought) possible between the two fiber types. The apparent connection of the two fiber types demonstrates that such multifiber type relationships are possible. These results, while not conclusive, do lend support to the concept that oxytalan fibers function in support of the tooth and/or components of the periodontal membrane. As shown by others, oxytalan fibers have been shown to relate closely to blood and lymphatic vessels found in the periodontal ligament (Carmichael 1968, Fullmer et al. 1974). These oxytalan fibers may thus help to stabilize these periodontal ligament elements, since they are closely related to collagen fibers, blood and lymphatic vessels.

It is possible that sectioning the teeth in $\mathrm{x}$-section would provide a more extensive view of collagen fibril oxytalan filament interweaving.

In conclusion, the following points should be considered:

(1) Oxytalan fibers relate closely to collagen. They interweave and may attach to collagen fibers.

(2) Oxytalan fibers attach to bone and cementum in some species.

(3) Oxytalan fibers function to stabilize the tooth and/or blood lymphatic vessels in the periodontal ligament.

\section{References}

Beertsen, W., Everts, V. \& VandenHoof, A. (1974) Fine structure of fibroblasts in periodontal ligament of the rat incisor and their possible role in tooth eruption. Archives of Oral Biology 19, 1087-1098.

Cameron, D. S., Jennings, E. H. \& Rannie, I. (1970) Oxytalan fibers in the human tympanic membrane. Journal of Laryngology and Otology $84,1235-1239$.

Carmichael, G. G. (1968) Observations with the light microscope on the distribution and connections of the oxytalan fibre of the lower jaw of the mouse. Archives of Oral Biology 13, $765-772$.

Carmichael, G. G. \& Fullmer, H. M. (1966) The fine structure of the oxytalan fiber. Journal of Cell Biology 28, 33-36.

Dawes, J. D. K. (1970) The aetiology and sequelae of exudative otitis media. Journal of Laryngology and Otology 84, 583-610.

Edwards, J. G. (1968) A study of the periodontium during orthodontic rotation of teeth. American Journal of Orthodontics 54, 441-461.

Fullmer, H. M. (1958) Differential staining of connective tissue fibers in areas of stress. Science $127,1240$.

Fullmer, H. M. (1959) Observations on the development of oxytalan fibers in peridontium of man. Journal of Dental Research 38, 510-518. 
Fullmer, H. M. (1960) Observations on the development of oxytalan fibers in dental granulomas and radicular cysts. Archives of Pathology 70, 59-67.

Fullmer, H. M. (1961) A histochemical study of periodontal disease in maxillary alveolar processes of 135 autopsies. Journal of Periodontology 32, 206-218.

Fullmer, H. M. (1962) Structural factors in periodontal disease. A critique of normal connective tissue of periodontium and some alterations with periodontal disease. Journal of Dental Research 41, 223-229.

Fullmer, H. M. \& Lillie, R. D. (1958) The oxytalan fiber: a previously undescribed connective tissue fiber. Journal of Histochemistry and Cytochemistry 6, 425-430.

Fullmer, H. M., Sheetz, J. H. \& Narkates, A. J. (1974) Oxytalan connective tissue fibers: a review. Journal of Oral Pathology 3, 291-316.

Fullmer, H. M. \& Witte, W. E. (1962) A histochemical study of periodontal membrane affected by scleroderma. Archives of Pathology $73,184-189$.

Goggins, J. F. (1966) The distribution of oxytalan connective tissue fibers in periodontal ligaments of deciduous teeth. Periodontics 4, 182-186.

Mander, S. T. K., Mander, L. N. \& Carmichael, G. G. (1968) The staining mechanism of aldehydefuchsin with reference to the oxytalan fiber in the mouse. Journal of Histochemistry and Cytochemistry 10(7), 480-485.

Miles, A. E. W. (1967- Structural and Chemical Organization of Teeth, Vol. 2, pp. 349-414. New York and London: Academic Press.

Provenza, D. V. (1965) Oral Histology, Inheritance and Development, pp. 497-529. Philadelphia: Lippencott.

Provenza, D. V., Fischlschweiger, W. \& Sisca, R. F.
(1967) Fibers in human dental papillae: a preliminary report on the fine structure. Archives of Oral Biology 12, 1533-1539.

Rannie, I. (1963) Observations on the oxytalan fibre of the periodontal membrane. Transactions of European Orthodontic Society 39, 127-136.

Ross, R. \& Bornstein, P. (1969). The elastic fiber. I. The separation and partial characterization of its macromolecular components. Journal of Cell Biology 40, 366-381.

Shackleford, J. M. (1974) The indifferent fiber plexus and its relationship to principal fibers of the periodontium. American Journal of Anatomy $131,427-442$.

Sheetz, J. H., Fullmer, H. M. \& Narkates, A. J. (1973) Oxytalan fibers: identification of the same fiber by light and electron microscopy. Journal of Oral Pathology 2, 254-263.

Sims, M. R. (1973) Oxytalan fiber system in the mouse mandible. Journal of Dental Research 52, 792-802.

Sims, M. R. (1977). The oxytalan fiber system in the mandibular periodontal ligament of the lathyritic mouse. Journal of Oral Pathology 6, 233-250.

Tedeschi, L. G. \& Sommers, S. C. (1961) Oxytalan fibers in sclerosing hemangiomas. Archives of Dermatology 84, 128-130.

Tedeschi, L. G. \& Sommers, S. C. (1962) Oxytalan fibers in sclerosing hemangiomas. Archives of sheath tumors. Archives of Dermatology 85 , $527-529$.

Address:

C. F. Cox

Laboratory of Oral Histology

Dental Research Institute

University of Michigan

Ann Arbor, Michigan 48109

U.S.A. 
This document is a scanned copy of a printed document. No warranty is given about the accuracy of the copy. Users should refer to the original published version of the material. 